\title{
VIII.
}

\section{Bericht über die Verhandlungen in der Section für Otologie und Laryngologie auf dem IX. Congresse polnischer Aerzte und Naturforscher in Krakau}

\author{
(21.-24. Juli 1900). \\ Von \\ Dr. R. Spira in Krakau.
}

Die erste Sitzung, Samstag den 21. Juli, war aussehliesslich der Laryngologie gwidmet.

Zweite Sitzung, zum Theil gemeinsam mit der chirurgisehen Section, Sonntag den 22. Juli Vormittags. Anwesende Mitglieder 70. Vorsitzende: Dr. Baracz (Lemberg) und Dr. Steiner (Warschau).

Guranowski (Warsehan) referirt tiber die Indicationen zur operativen Behandlung shroniseher Mittelohreiterungen.

$G$ uranowski theilt die Indicationen zur radicalen Operation ein, in 1. absolute Indicationen, welche vorhanden sind beim Auftreten intracranieller Complicationen und allgemeiner Erscheinungen, wie Pyämie und Septicämie, selbst bei äusserlich unverändertem Warzenfortsatze, ferner bei subjectiven und objectiven Entzinndungserscheinungen und Cholesteatom des Warzenfortsatzes; 2. relative Indicationen; zu ihnen gehören vor Allem Erscheinungen, welche darauf hinweisen, dass der pathologisehe Process die Grenzen der eigentlichen Paukenhöhle ïbersehritten hat, wie Senkung der hinteren Gehörgangswand, nach ihrer $\mathrm{Ab}$ tragung rasch nachschiessende Granulationen im hinteren $\mathrm{Ab}$ sohnitte der Paukenhöhle, Fisteln im hinteren Absehnitte des Gehörganges, nach seiner Entfernung raseh von hinten und oben nachfliessender Eiter, aus Atticus und Antrum sich entleerende Cholesteatommassen, Facialislähmung, ferner jugendliches Alter der Patienten, welches bekanntlich das grösste Contingent für intracranielle Complicationen liefert. Copiöse fötide Eiterung erweeken den Verdacht auf einen tiefer greifenden Process und bilden eine Mahnung, nicht zu lange mit der Operation zu 
Verhandlungen auf dem IX. Congresse polnischer Aerzte u. s. w. 131

zögern. 3. Contraindicirt ist die Radicaloperation bei diffuser eitriger Meningitis, malignen Neoplasmen des Felsenbeines und in Fällen weit vorgeschrittener Lungentuberculose.

Heimann (Warschau) referirt: Ueber die Indicationen zur chirurgisehen Behandlung chroniseher Mittelohreiterungen.

Die Behandlung dieser Affection soll im Anfang immer eine conservative sein, mit Ausnahme jener Fälle, wo ein unblutiges Verfahren erfolglos bleibt oder directe Indication zur ehirurgischen Intervention vorhanden ist. Die chronischen Eiterungen ans dem Ohr müssen principiell geheilt werden, daraus folgt, dass dort, wo das klinische Bild und die anatomischen Veränderungen eine Heilung auf conservativem Wege als unmöglich erscheinen lassen, es unzweckmässig und irrationell ist, ein nicht operatives Verfabren Monate und Jahre lang fortzusetzen. Als erste Etappe für die Behandlung von Obreiterungen gilt die Entfernung der Gehörknöchelchen durch den äusseren Gehörgang, was manchmal zur Heilung führen kann. Doch werden die Indieationen zu dieser Operation immer mebr eingeschränkt, da nur selten Caries der Knöchelchen allein besteht, vielmehr in den meisten Fällen gleichzeitig die umgebenden Knochenwände afficirt sind.

Die Discussionen über die Indicationen zur Radicaloperation haben bis jetzt noch zu keiner einheitlichen Fixirung derselben gefübrt. Wo drohende Symptome oder Complicationen seitens der Schädelhöhle bestehen, da ist die Indication zur Operation allgemein zugestanden. Diese ist aber auch nothwendig bei ChoJesteatom, bei Caries verschiedener Abschnitte des Schläfenbeines, bei in anderer Weise nicht rasch genug zu beseitigender Eiterretention, bei schmerzhafter Anschwellung und bei Fisteln am Warzenfortsatz, bei Senkungsabscessen und bei Tuberculose des Schläfenbeines. Wo diese Erscheinungen nicht deutlich ausgesprochen und nicht sicher diagnosticirt sind, besteht nur eine relative Indication zur Operation. Wo aber nur die Scbleimhaut der Paukenhöhlen allein Sitz der Eiterung ist, da ist die operative Behandlung direct contraindicirt.

Die Zeit zu bestimmen, wie lange man bei chronischen Eiterungen, die nicht unbeding,t eine sofortige chirurgische Intervention erfordern, mit der Operation warten kann, muss der Erfahrung des Arztes überlassen werden und ist vorzüglich von dem otoskopischen Bilde abhängig.

Krajewski (Warschau) findet, dass man in der Geschichte dieser Operation drei Perioden unterscheiden kann, die erste 
mit einem am meisten ausgedehnten, die zweite mit einem bis auf ein Minimum eingeschränkten Indicationsgebiet; die jetzt beginnende, dritte Periode sollte sich durch Ausgleichung dieser beiden extremen Richtungen auszeichnen. Krajewski ist nur für absolute, streng zu definirende Indicationen.

Barącz (Lemberg) erwähnt, dass Referent der die Eiterung unterbaltenden Perforation der Shrapnell'schen Membran Erwähnung zu thun vergessen hat. Die Eintheilung der Indicationen. durch Guranowski in absolute und relative hält er für richtig.

Spira (Krakau) meint, es werde zu oft operirt. Die blosse, wenn auch hartnäckige Eiterung berechtigt nicht zur Operation, auch nicht aus prophylaktischen Gründen, und zwar umsoweniger, als auch die sogenannte Radicaloperation nicht immer sicher die Eiterung zur Heilung bringt und nicht einmal sicher den gefährlichen Complicationen vorbeugen kann. Er erkennt nur die absoluten Indicationen als gerechtfertigt an.

Ka der (Krakau) verwendet mit gutem Erfolge einen Lappenschnitt mit nach oben gerichteter Basis.

Guranowski versteht unter absoluter Indication, Indicatio vitalis. Man kann nicht behaupten, dass eine Perforation in der Shrapnell'sehen Membran immer die Eiterung unterhalte.

Dritte Sitzung Montag, den 23. Juli Nachmittags.

VII. Spira (Krakau), Ueber die Pathogenese der functionellen Labyrinthersehütterung (Commotio labyrinthi).

Das Zustandekommen der Labyrintherscheinungen nach einem Trauma in jenen Fällen, in welchen das Trauma keine anatomischen Veränderungen verursacht hat, sucht Vortragender nach Anführung der Theorien anderer Autoren in der Weise zu erklären, dass er annimmt, durch die Erschütterung werde der Zusammenhang der zelligen Bestandtheile, aus denen die Neurone des Hörnervenapparates sich zusammensetzen, gelockert, wodurch ihre Leistungsfähigkeit herabgesetzt, ihre Empfindungsschwelle verschoben werde. Die Verschiebungen können sich im Gebiete der Vorhofsnerven, als des statischen Apparates rasch wieder ausgleichen. Die hierher gehörigen Neurone kehren bald wieder in ihre Gleiehgewiehtslage zurüek, ja sie können sich sogar an oft sich wiederholende Reize derart gewöhnen, dass sie schliesslich von ihnen unberührt blieben. Daher der anfangs bei der Seekrankheit, bei Tänzen, beim Schankeln, Carousselfahren, bei Dachdeckern und Kaminfegern $\mathfrak{u}$. s. w. auftretende Schwindel mit der Zeit immer schwächer wird und mit der Gewöhnung 
ganz verschwindet. Anders verbalten sich die Neurone im Gebiete des N. cochlearis, die einmal aus ihrer ursprünglichen Lage verschoben, nicht mehr so leicht in dieselbe zurïckkehren, im Gegentheil, sich bei Fortdauer oder ofterer Wiederholung des spectidechen Reizes (Schall) immer mehr von der Ruhelage entfernen: Daher finden wir bei Personen, die der Einwirkung solch Keize öfters ausgesetzt sind, bleibende Störungen (Ohrenrausheñ, progressive Schwerhörigkeit u. s. w.), wie bei Maschinisten, 业esselschmieden, Artilleristen $\mathrm{u}$. dgl.

Die Wirkung der Hörübungen bei Schwerhörigkeit sucht Spira in analoger Weise zu erklären, wie den Einfluss der gymnastischen Uebungen auf die Nerven bei peripheren und centralen Nervenlähmungen. Beide Arten von Uebangen wirken 1. einerseits "bahnend", wodurch Nerven, die durch irgend einen kranklhaften Process ihre Leistungsfähigkeit verloren haben, ihre Funetionsfähigkeit wieder erlangen und für die specifischen Reize wieder empfindlich werden können. 2. andrerseits „compensirend“, wodurch ein noch functionstüchtiger Rest des erkrankten nervösen Sinnesorganes die Fähigkeit erlangen kann, den inactiven Theil dieses Organes theilweise wenigstens zu compensiren, für ihn vicariirend einzutreten.

Die Idiosynkrasie, vermöge welcher manche Personen bei gewissen Bewegungen, Tänzen, Fahrt auf der Bahn u. dgl. von Uebelkeit und Schwindel befallen werden, beruht nach Spira auf einer excessiven Erregbarkeit des statischen Organes, des Ampullenapparates. Um diesem Uebel abzuhelfen, schlägt er eine Reihe gymnastischer Uebungen vor, welche den Zweck haben, durch Angewöhnung an Bewegungen in der Richtung: aller drei Dimensionen die Abstumpfung der Empfindlickeit und die Steigerung der Widerstandsfäligkeit der entsprechenden Centren und Nervenendigungen des statischen Apparates gegen die bezüglichen äusseren Sehädlichkeiten zu erzielen.

\section{Discussion.}

Za lew ski (Lemberg) behauptet, dass wegen Mangels einer Controlle durch anatomische Untersuchungen das Fehlen einer anatomiseben Basis bei Labyrintherschütterung nicht mit Sicherheit angenommen werden kann; er erachtet daher die Diagnose dieser Krankheit fïr unmöglich.

H e imann vermuthet für alle Fälle von Commotio labyrinthi organische Veränderungen in diesem Organe. Der Schwindel 
bei Schiffs- und Bahnfahrten, Tänzen u. s. w. sei eher auf Störungen im Kleinhirn zu beziehen.

Bau rowiez (Krakau) sehliesst sich dieser letzteren Ansicht Heimann's an.

Gura now ski (Warschau) hält gleichfalls den Mangel einer anatomischen Veränderung für unwahrscheinlich und macht aufmerksam auf die besondere Vorsicht, die nothwendig ist bei einem Kranken, der über Erschütterungserscheinungen klagt und ein ärztliches Zeugniss verlangt, welches ihm die Krankheit bestätigen soll. Spira beruft sich auf Fälle mit den entsprechenden Erscheinungen in vivo und negativem Obductionsbefund post mortem und auf die analoge Affection der Gehirnerschütterung.

VIII. Spira, Anträge zur Verhütung von Taubheit und Taubstummheit.

Gestützt auf casuistische und statistische Daten in der Literatur weist Spira nach, dass 1 . in Taubstummen-Anstalten sich nicht selten Zöglinge finden, die mit verschiedenen Ohrenkrankheiten behaftet sind, durch deren zweekmässige Behandlung auf die Hörfähigkeit günstig eingewirkt werden könnte, 2. Schulkinder oft mit Affectionen der oberen Luftwege, adenoiden Vegetationen, verschiedenen Erkrankungen des Gehörganges behaftet sind, welche dureb Uebersehen und Nachlässigkeit zur Taubheit und Taubstummheit führen und durch deren rechtzeitige Berücksichtigung diesem Uebel vorgebeugt werden könnte. Am Schlusse seiner Ausfülirungen legt Spira der Section folgenden Antrag zur Beschlussfassung vor:

"Der IX. Congress polnischer Aerzte und Naturforscher fordert die entsprechenden Behörden auf zu verfügen:

1. Eine Zusammenstellung statistisoher Daten, betreffend die Zahl der Tauben und Taubstummen im Lande, die Untersuchung des gegenwärtigen Zustandes ihres Gehörorgans und der Ursachen dieses Gebrechens bei den Zöglingen der TaubstummenAnstalten.

2. Dureh die Sehul- und Sanitätsbehörden vorzunehmende systematische Untersuchungen sämmtlicher Schulkinder in Bezug auf ihre Hörschärfe, und die Unterwerfung ohrkranker Kinder einer sachgemässen, wissenschaftlichen Behandlung."

Nach einer kurzen Discussion wurden diese Anträge einstimmig und mit Anerkennung angenommen. In der Schlusssitzung des Congresses wurden sie später zum Beschlusse erhoben. 
IX. Baurowicz (Krakan), Ueber die Operation an der unteren Nasenmusehel.

Unabhängig von 0 stmann und von dessen Publicationen im Arehive für Laryngologie hat Baurowicz die nene Operationsmethode ausgeubt, welehe darauf beruht, eine tiefe Furche an der oberen Muschelfäche mit dem Thermokauter auszubrennen, um den Rest der hypertrophisehen Partien bequem mit der Schlinge oder der Schere abtragen zu können. Die ihn leitende Idee war nicht Bequemlichkeit, sondern die unblutige Operation, indem dureh die Furche die Continuität der Gefässe unterbrochen und so die Beendigung der ganzen Operation mit der Beckmann'schen Sehere und der kalten Sehlinge in einer Sitzung ermöglicht wird, ohne dass die Austamponirung der Nasenhöhle nöthig wäre; eine eventuelle geringe Blutung kann dann durch eine leichte Tamponade zum Stillstande gebracht werden. Baurowiez verwirft den Elektrokanter und zieht den blutigen Weg vor; in entsprechenden, ziemlich häufigen Fällen besehränkt er sich auf die Abtragung des hinteren hypertrophisehen Muschelendes. In Fällen von Sehwellung der Sehleimhaut übt er die Massage derselben, die, wenngleich erst nach langer Behandlung, von günstigem Erfolge begleitet zu sein 'pflegt. Baurowioz warnt vor allzu voreiliger Abtragung der Sehleimhant, welehe nachträgliehe Austroeknung der Nase, besonders bei (serophulösen) Personen mit bereits bestehender Trookenheit der Pharynxschleimhaut, nach sich ziehen kann. 